\title{
Evidencia de validez en Chile del Alcohol, Smoking and Substance Involvement Screening Test (ASSIST)
}

\section{Validity evidence of the Alcohol, Smoking and Substance Involvement Screening Test (ASSIST) in Chile}

\author{
Gonzalo Soto-Brandt*, Rodrigo Portilla Huidobro**, David Huepe Artigas***, Álvaro Rivera- \\ Rei***, María Josefina Escobar***, Natalia Salas Guzmán****, Andrés Canales-Johnson***. \\ Agustín Ibáñez***, Claudio Martínez Guzmán*****, Álvaro Castillo-Carniglia* \\ *Área de Estudios, Servicio Nacional para la Prevención y Rehabilitación del Consumo de Drogas y Alcohol (SENDA), Chile; \\ **Área de Tratamiento, Servicio Nacional para la Prevención y Rehabilitación del Consumo de Drogas y Alcohol (SENDA), \\ Chile; ***Núcleo UDP-Fundación INECO para las Neurociencias (NUFIN), Universidad Diego Portales, Santiago, Chile; \\ ****Facultad de Educación, Universidad Diego Portales, Santiago, Chile; *****Facultad de Psicología, Universidad Diego \\ Portales, Santiago, Chile.
}

\section{Resumen}

El objetivo del estudio fue analizar la validez de la versión chilena de un instrumento para la identificación de diferentes niveles de riesgo asociado al consumo de alcohol, tabaco, marihuana y cocaína (Alcohol, Smoking and Substance Involvement Screening Test, ASSIST). Se evaluó la fiabilidad y consistencia del instrumento además de la validez concurrente y discriminante. La muestra fue de 400 usuarios de servicios de tratamiento drogas y alcohol ambulatorios y residenciales de la atención primaria de salud, comisarías y empresas. La consistencia interna obtenida fue alta (Alcohol $\alpha=.86$. marihuana $\alpha=.84$ y cocaína $\alpha=.90)$. El coeficiente de correlación intraclase (CCI) con la comparación test-retest fue buena para Alcohol $(\mathrm{CCI}=.66)$, marihuana $(\mathrm{CCI}=.74)$ y cocaína $(\mathrm{CCI}=.80)$. Se observó una buena correlación entre los puntajes del ASSIST el puntaje del AUDIT ( $r$ de Pearson $=.85)$, ASI-Lite $(r$ entre .66 y .83 para tabaco, alcohol, marihuana y cocaína) y SDS $(r=.65)$. El punto de corte original para la detección del riesgo alto es de 27 puntos, sin embargo, considerando un mejor balance entre sensibilidad y especificidad se modificó ese corte a 21. Los resultados obtenidos en éste estudio demostraron las buenas propiedades psicométricas del ASSIST para la detección de distintos niveles de riesgo asociados al consumo de sustancias en población general de Chile.

Palabras Clave: alcohol, drogas ilícitas, tabaco, cribado, evidencia de validez.

\begin{abstract}
This study aims to psychometrically validate the Chilean version of the Alcohol, Smoking and Substance Involvement Screening Test ASSIST. Specifically, this study is interested in evaluating the reliability, consistency and concurrent and discriminant validity of this instrument. The sample was composed for a total of 400 people from four different settings: treatment centers (residential and ambulatories), primary health care, police stations and companies. The reliability of the ASSIST was high $(\alpha=.86$ for Alcohol, $\alpha=.84$ for marijuana and $\alpha=.90$ for cocaine). The intra class correlation coefficient (ICC) with test-retest comparison was statistically significant for Alcohol $(\mathrm{ICC}=.66)$, marijuana $(\mathrm{ICC}=.74)$ and cocaine $(\mathrm{ICC}=$ $.80)$. There were statistically significant correlations between the ASSIST and the AUDIT score (Pearson's $r=.85$ ), the ASSIST and the ASI-Lite score ( $r$ between .66 and .83 for tobacco, alcohol, marijuana and cocaine), and the ASSIST and the SDS score $(r=.65)$. The original cutoff point for high risk detection was 27 points, however, in order to have a better balance between sensitivity and specificity the cut was changed to 21 points. The ASSIST presents good psychometric properties and therefore is a reliable and valid instrument to be used as a mechanism to detect risk levels of substance use in the Chilean population.

Key Words: alcohol, illicit drugs, smoke, screening test, validation.
\end{abstract}


$\mathrm{E}$ 1 consumo de alcohol y otras drogas son un importante causa morbimortalidad a nivel global y su impacto genera costos sanitarios, sociales y económicos. La tasa de mortalidad atribuible al consumo de alcohol y otras drogas ilícitas es de aproximadamente 39 por cada 100,000 habitantes a nivel mundial, de las cuales el $90 \%$ son por alcohol. Asimismo, es responsables de la pérdida de 13 años de vida perdidos ajustados por discapacidad (AVAD) por cada 1,000 habitantes en el mundo (World Health Organization, 2010).

En Chile, según el último estudio nacional de drogas en población general el año 2012 un $7.3 \%$ de la población de 12 a 64 años declara haber consumido alguna droga ilícita (marihuana, clorhidrato de cocaína o pasta base) en el último año, de los cuales un $28.1 \%$ presenta un probable abuso y/o dependencia (Servicio Nacional para la Prevención y Rehabilitación del consumo de Drogas y Alcohol, 2013). Los costos económicos asociados al consumo de drogas se han estimado en aproximadamente 500 millones de dólares al año, equivalente al $0.45 \%$ del producto interno bruto del año 2006 (Fernández, 2012). Por su parte, en un estudio publicado recientemente se estimó que el 9.8\% del total de muertes ocurridas en la población de 15 años y más en Chile son atribuibles al consumo de alcohol (Castillo-Carniglia, Kaufman y Pino, 2013).

La adecuada planificación y administración de servicios y programas de prevención y tratamiento requieren una comprensión clara de la naturaleza y el alcance de las necesidades del individuo. Los instrumentos de cribado son fundamentales para la detección temprana del consumo y abuso de sustancias, áreas problemáticas y otros indicadores que reflejan la necesidad de atención de los servicios de tratamiento y atención de salud (Drug Misuse Information Scotland, 2000). No obstante, existen limitaciones en el empleo de pruebas de cribado debido al excesivo tiempo de aplicación, su extensión y a la complejidad de sus cálculos (Babor y Kadden, 2005; Humeniuk et al., 2008).

Ante la necesidad de contar con un instrumento de cribado de rápida administración, simple en sus cálculos y que logre detectar usuarios con consumo riesgoso y abusivo de alcohol, la Organización Mundial de la Salud (OMS) diseñó una prueba de cribado denominada Alcohol Use Disorders Identification Test (AUDIT) la cual ha demostrado abundante evidencia de validez y fiabilidad medida en numerosos estudios (Alvarado, Garmendia, Acuña, Santis y Arteaga, 2009; Allen, Litten, Fertig y Babor, 1997; Saunders, Aasland, Babor, de la Fuente y Grant, 1993). Posteriormente, la OMS desarrolló un programa para la elaboración de un nuevo instrumento de cribado que además permitiese pesquisar el consumo del tabaco y drogas ilícitas, y que sea fácilmente vinculable a una intervención breve en contextos de servicios de atención primaria de salud, atención médica general y otros entornos. Este instrumento se denominó ASSIST por sus siglas en inglés (Alcohol, Smoking and Substance Involvement Screening
Test) y fue validado con un estudio multicéntrico realizado en Australia, Brasil, India, Tailandia, Reino Unido, Zimbabue y Estados Unidos (WHO ASSIST Working, 2002; Humeniuk et al., 2008; Organización Mundial de la Salud, 2011). Este estudio consideró un muestra de participantes en tratamiento por consumo de drogas y alcohol en la red de salud y población consumidora pero sin un diagnóstico clínico de abuso o dependencia (WHO ASSIST Working Group, 2002). Este y otros estudios han demostrado validez y fiabilidad del ASSIST en distintos ámbitos y poblaciones, entre ellos el contexto escolar y en poblaciones especificas tales como adultos mayores y embarazadas (Henrique, De Micheli, Lacerda, Lacerda y Formigoni, 2004; Hotham, 2009; Humeniuk et al., 2008; R Khan et al., 2011; Newcombe, Humeniuk y Ali, 2005).

El ASSIST, en su versión 3.0, es un cuestionario de ocho preguntas de aplicación breve que evalúa riesgos para la salud y otros problemas asociados al consumo de sustancias. El instrumento indaga respecto a la frecuencia y patrones de uso de sustancias y arroja como resultados el nivel de riesgo asociado al consumo el que a su vez se vincula a una intervención o a una estrategia de derivación.

El sistema de salud chileno es mixto, está compuesto por un sector privado que concentra a las personas con menores riesgos de salud y con un seguro individual de financiamiento, y un sector público que atiende a las personas con mayores riesgos de salud y con un seguro solidario de financiamiento (Larrañaga, 1997). El sector público atiende más de 13 millones de personas que constituye cerca del $75 \%$ de la población nacional (Fondo Nacional de Salud, 2013). E1 90\% de las personas atendidas en el sector público lo hace en la Atención Primaria de Salud (Departamento de Estadísticas e Información de Salud, 2013), por lo que su amplia cobertura la convierte en un excelente ámbito para la utilización de pruebas de cribado (Coulton et al., 2006; Watson et al., 2013).

Por lo anterior, resulta relevante contar con instrumentos breves, válidos y confiables que consideren las particularidades lingüísticas y tipos de sustancias usadas en el cada país. Esto permitirá la planificación e implementación de una política pública de detección temprana, intervención breve y referencia a tratamiento que contribuya a mejorar la salud de la población.

El objetivo del presente estudio fue analizar la validez del instrumento ASSIST para la identificación del riesgo asociado al consumo de drogas en población adulta chilena de diferentes ámbitos (centros de atención primaria de salud, centros de tratamiento y rehabilitación de drogas y alcohol, población laboral y personas detenidas en comisarías policiales).

\section{Método}

\section{Participantes}

Se recurrió a una muestra no probabilística por accesibilidad, contemplando los siguientes criterios de exclusión: 
(i) presencia de un estado agudo de intoxicación, (ii) trastorno psiquiátrico grave con síntomas al momento de la entrevista (síntomas psicóticos. alteraciones del estado de conciencia, agitaciones psicomotoras con necesidad de contención, síntomas de abstinencia graves), (iii) deficiencia cognitiva severa, y (iv) de edad menor a 18 años. La muestra seleccionada buscó incluir población con diferentes niveles y patrones de uso de drogas de modo aumentar las posibilidades de encontrar participantes en los rangos de puntaje bajos, medio y altos.

El total de participantes encuestados fueron 400 participantes provenientes de los sistemas de salud primario de Santiago de Chile, centros de tratamiento de drogas, ámbito laboral, y detenidos en comisarías. No se ofrecieron incentivos económicos ni materiales a los entrevistados. El estudio no fue evaluado por un comité de ética, aunque sí contó con la revisión y aprobación por escrito de las autoridades máximas de los Servicios de Salud, direcciones Municipales de Salud y direcciones de los Centros de Atención y Comisarías donde se llevó a cabo el estudio. Asimismo, todos los participantes leyeron y firmaron un consentimiento para participar en el estudio (ver apéndice).

\section{Adaptación lingüística}

La primera etapa realizada fue la adaptación lingüística del instrumento ASSIST V3.0 en español de la OMS al idioma español de uso en Chile. Este procedimiento tuvo por objetivo asegurar la correspondencia semántica y de contenido entre la versión chilena y su original en español e inglés (OMS). En primer lugar se hizo una adaptación considerando la experiencia clínica de los profesionales del equipo investigador, adaptando principalmente los nombres y ejemplos de las sustancias. Luego se convocó a un comité de expertos compuesto por psiquiatras, psicólogos e investigadores con experiencia en la temática de alcohol y drogas para evaluar si, en el contexto chileno, las preguntas del ASSIST parecían relevantes, razonables, claras y no ambiguas. Se consensuó una primera versión del cuestionario.

Se aplicó la versión adaptada al contexto chileno a una muestra de 20 pacientes en tratamiento y tras evaluar su adaptación lingüística y semántica del instrumento, se volvió a convocar al comité de expertos quienes entregaron sus recomendaciones finales y acordaron la versión que sería utilizada en el presente estudio (ver apéndice).

\section{Instrumentos}

Además de un breve cuestionario sociodemográfico se describen a continuación la batería de instrumentos utilizados dentro del proceso de validación:

La prueba de detección ASSIST 3.0 evalúa el nivel de riesgo asociado al uso de drogas a partir de 8 ítems que exploran seis áreas: uso de sustancias a lo largo de la vida, uso de sustancias durante los últimos tres meses, problemas relacionados con el consumo, riesgo actual de presentar problemas en el futuro, posibilidad de dependencia y uso de drogas por vía intravenosa. La versión 3.0 abarca diez sustancias: tabaco, alcohol, cannabis, cocaína (en el caso chileno, incluye pasta base y clorhidrato de cocaína), anfetaminas, inhalantes, sedantes, alucinógenos, opiáceos y otras drogas. Las puntuaciones finales para cada sustancia son clasificadas en tres niveles de riesgo (bajo, intermedio y alto) que sirven para orientar al profesional en cuanto a la intervención más adecuada para cada caso, y dado que es un instrumento breve y de fácil manejo puede ser utilizado en diversos escenarios y por personal no especializado. $\mathrm{Su}$ aplicación toma entre 5 y 15 minutos dependiendo del número de sustancias que consume cada usuario.

La descripción de cada ítem y la fórmula del cálculo de los puntajes se detalla en el manual de aplicación de la OMS (2011).

Los puntajes utilizados para la validación fueron: (i) puntaje especifico por sustancia a través de sumatoria de los ítems dos al siete, (ii) puntaje total con la sumatoria de los ítems uno al ocho para cada sustancia, (iii) prevalencia de consumo en los últimos tres meses a partir del primer ítem del instrumento para cada sustancia, (iv) puntaje para identificación de dependencia a través de la sumatoria los ítems 1, 2, 3, 6, y 7 para cada sustancia, y (v) puntaje para identificación de abuso a través de la sumatoria los ítems 1, 2, 4, 5 y 6 para cada sustancia (Newcombe et al.. 2005).

Para medir la severidad del consumo de sustancias se utilizó el instrumento Addiction Severity Index (ASI-Lite) que tiene 22 preguntas con distintas escalas de respuestas que indagan sobre el uso de alcohol y drogas en la vida, en los últimos 90 días y problemas asociados al consumo. También aborda la historia familiar del consumo de sustancias. Distintas validaciones han demostrado sus adecuadas propiedades psicométricas tanto en su versión en inglés (Cacciola, Alterman, McLellan, Lin y Lynch, 2007; McLellan, Luborsky, Woody y O’Brien, 1980), en su adaptación en idioma español (Diaz et al., 2010) y su versión completa validada en Chile (Consejo Nacional para el Control de Estupefacientes, 2008). La consistencia interna del cuestionario observada en la muestra fue adecuada $(\alpha=.75)$ para el conjunto de los ítems de las sustancias principales (Tabaco, alcohol, cocaína y marihuana).

El instrumento Severity of Dependence Scale (SDS) se utilizó para medir la severidad de la dependencia a alguna droga. Tiene cinco ítems que cuantifican el efecto del consumo de drogas en la vida; cada ítem tiene respuesta tipo Likert de cero a cuatro. Sus validaciones han demostrado sus capacidades psicométricas en su versión en inglés (Gossop, Best, Marsden y Strang, 1997; Gossop et al., 1995) y su adaptación en idioma español (Gonzalez-Saiz, de Las Cuevas, Barrio y Domingo-Salvany, 2008; Iraurgi, González, Lozano, Landabaso y Jiménez, 2010; Iraurgi, Lozano, González-Saiz y Trujols, 2008). La consistencia interna de los cinco ítems del cuestionario observada en la muestra fue alta $(\alpha=.90)$. 
El instrumento Mini-International Neuropsychiatric Interview (MINI-Plus) se utilizó para el diagnóstico de trastornos por consumo de alcohol y de otras sustancias (Marihuana y cocaína); en total 23 ítems con respuestas binarias. Sus validaciones han demostrado sus capacidades psicométricas en su versión original en inglés (Lecrubier et al., 1997; Sheehan et al., 1998) y su adaptación en idioma español (Bobes, 1998). La consistencia interna de los ítems del cuestionario fue alta $(\alpha=.97)$.

Para la detección y manejo de los problemas relacionados al alcohol se utilizó el cuestionario de auto registro AUDIT, que tiene en total diez ítems con puntaje entre cero y cuatro puntos. Sus validaciones han demostrado sus capacidades psicométricas en su versión original en inglés (Bohn, Babor y Kranzler, 1995; Saunders et al., 1993), su adaptación en idioma español (Rubio, Bermejo, Caballero y Santo-Domingo, 1998), y su validación en Chile (Alvarado et al., 2009). La consistencia interna de los diez ítems del cuestionario en el presente estudio fue alta $(\alpha=.93)$.

Finalmente, se utilizó el instrumento Revised Fagerstrom Tolerance Questionnaire Smoking (RTQ) para valorar la severidad por el consumo de nicotina, el cual consta de diez ítems con escala de respuesta estilo Likert que va de uno a cinco. El instrumento presenta validación en su versión revisada (Tate y Schmitz, 1993) y su versión en español (Clemente et al., 2003). La consistencia interna de los diez ítems del cuestionario en la muestra del presente estudio fue alta ( $\alpha$ $=.80)$.

Adicionalmente se realizaron entrevistas clínicas complementarias para los análisis de la validez discriminante.

\section{Entrevistas Clínicas}

Las entrevistas fueron realizadas a personas asistentes a un programa de tratamiento y tenían por objetivo indagar respectos del consumo de drogas siguiendo los criterios del DSM-IV-TR para dependencia y abuso de substancias (American Psychiatric Association, 2000). Con esta exploración clínica, el psicólogo generó una calificación respecto si el paciente cumple o no con los criterios de abuso o dependencia a alguna sustancia. Finalmente, el entrevistador se pronunció acerca de la gravedad de consumo del paciente desde su impresión clínica, marcando un instrumento de medida tipo Likert de siete puntos, donde uno indica menor gravedad y siete máxima gravedad.

\section{Procedimiento}

Para la aplicación de los instrumentos se dispuso de dos equipos de psicólogos y licenciados en psicología que estuvieron bajo la supervisión de un coordinador de terreno y un psicólogo clínico. Ambos equipos recibieron una capacitación respecto a la aplicación de los instrumentos incluidos en el estudio. Asimismo los entrevistadores fueron entrenados en la evaluación clínica de trastornos por consumo de sustancias según los criterios DSM-IV-TR. Todas las entrevistas fueron realizadas en los lugares de procedencia (centros de tratamiento de drogas en quienes estaban en tratamiento, centros de salud primaria, lugares de trabajo y comisarias) en una sala sin la presencia de terceras personas con el objetivo de asegurar la confidencialidad de la información entregada.

En la primera medición se aplicó el ASSIST y la batería de instrumentos a 400 participantes, 75 de ellos fueron evaluados con entrevistas clínicas para tener una medida de validez externa. Catorce días después se realizó una segunda medición a 93 participantes que consideró sólo el ASSIST.

\section{Análisis estadístico}

Se calculó el alpha de Cronbach para evaluar la consistencia interna del ASSIST con el programa FACTOR 9.2 (Lorenzo-Seva y Ferrando, 2006) que permite el cálculo para datos ordinales. Para el análisis de fiabilidad test-retest se utilizó el coeficiente de correlación intraclase (CCI), y el criterio considerado para su valoración como bueno fue de .60 o más y como excelente de.75 o más (Cicchetti y Sparrow, 1981). La validez concurrente del ASSIST y los cinco instrumentos escritos anteriormente (ASI-Lite, SDS, MINI-Plus, AUDIT, RTQ-Smoking) se analizó a través de correlación de Pearson y t-test para muestras dependientes. El criterio considerado para la valoración de la correlación como media fue mayor a .40 , alta mayor a $.60 \mathrm{y}$ muy alta mayor a .80 (Hart, 2007).

Para explorar la estructura interna de la prueba de medida ASSIST se realizó un análisis factorial exploratorio especificando una matriz de correlación policórica. Finalmente, y para determinar los puntos de corte del instrumento se utilizó el análisis de la curva característica de operación del receptor (ROC) que permitió determinar la sensibilidad, o capacidad de la prueba para detectar a personas con algún nivel de riesgo asociado al consumo, y especificidad, o capacidad de la prueba para detectar a personas sin riesgo. Este análisis se realizó para determinar las categorías de riesgo intermedio y riesgo alto. Como referencia para la categoría de riesgo alto se utilizó el diagnóstico derivado de la entrevista clínica; para la categoría de riesgo intermedio con un consumo de tres o más días en los últimos tres meses y; riesgo bajo si no hay consumo o si éste es menor a tres días en los últimos tres meses.

Todo los análisis psicométricos de realizaron por separado para tabaco, alcohol, marihuana y cocaína. La exclusión de las demás sustancias responde a la baja prevalencia de consumo en la muestra. No se realizó ningún procedimiento de imputación de valores perdidos. En la primera entrevista la totalidad de los entrevistados respondieron el ASSIST, sin embargo de los 100 participantes considerados para el retest, 7 no pudieron ser contactados. Los análisis se realizaron con el software Stata 11.2 (StataCorp, 2009) y la significancia estadística considerada en los análisis fue $p$ $<.05$. 


\section{Resultados}

La muestra $(n=400)$ estuvo constituida por 211 participantes de centros de atención primaria, 97 participantes de centros de tratamiento de drogas (Ambulatorios y residenciales), 73 participantes del ámbito laboral y 19 participantes del ámbito policial (comisarías). El 56\% fueron mujeres y la media de edad fue de 32.9 años con una desviación estándar de 10.1. El 45\% de los participantes se encontraban solteros, mientras que un $50.7 \%$ informó contar con trabajo. El $72 \%$ reportó tener educación secundaria completa o superior. En la tabla 1 se muestra el reporte de consumo de las sustancias principales en los último tres meses y su distribución según aspectos socio demográficos.

En la tabla 2 se presenta la descripción en términos de su media, desviación estándar, asimetría y curtosis de los siete primero ítems del ASSIST y del puntaje total obtenido a partir de la sumatoria del ítem 2 al 7 para tabaco, alcohol, ma-

Tabla 1

Prevalencia de consumo de sustancias según aspectos socio demográficos y procedencia (\%)

\begin{tabular}{|c|c|c|c|c|}
\hline & Tabaco & Alcohol & Marihuana & Cocaína \\
\hline Total muestra & 58.8 & 74.3 & 25.0 & 21.3 \\
\hline \multicolumn{5}{|l|}{ Sexo } \\
\hline Hombre & 61.9 & 87.5 & 34.1 & 31.8 \\
\hline Mujer & 56.3 & 63.8 & 17.9 & 13.0 \\
\hline \multicolumn{5}{|l|}{ Edad } \\
\hline $18-25$ años & 59.3 & 69.4 & 36.1 & 17.6 \\
\hline 26-31 años & 62.0 & 79.4 & 28.3 & 23.9 \\
\hline $32-40$ años & 58.3 & 79.6 & 24.1 & 30.6 \\
\hline 41 y más años & 54.4 & 68.9 & 10.0 & 12.2 \\
\hline \multicolumn{5}{|l|}{ Nivel educacional } \\
\hline Básica incompleta/completa & 68.5 & 72.2 & 25.9 & 27.8 \\
\hline Media incompleta/completa & 61.4 & 75.0 & 28.4 & 21.6 \\
\hline Técnica incompleta/completa & 61.1 & 74.4 & 20.0 & 24.4 \\
\hline Universitaria incompleta/completa & 43.6 & 74.4 & 23.1 & 12.8 \\
\hline Sin estudios & 50.0 & 50.0 & 0.0 & 0.0 \\
\hline \multicolumn{5}{|l|}{ Estado civil } \\
\hline Casado(a)/convive & 49.7 & 70.9 & 16.4 & 12.2 \\
\hline Divorciado(a)/separado(a) & 71.0 & 74.2 & 25.8 & 38.7 \\
\hline Solterolal & 66.1 & 77.8 & 33.9 & 27.8 \\
\hline \multicolumn{5}{|l|}{ Situación Ocupacional } \\
\hline Empleadolal & 59.9 & 79.9 & 23.9 & 18.5 \\
\hline Desempleadolal & 66.7 & 81.5 & 22.2 & 18.5 \\
\hline Inactivolal & 53.6 & 59.1 & 27.3 & 28.2 \\
\hline \multicolumn{5}{|l|}{ Procedencia } \\
\hline Centros de atención primaria de salud & 55.2 & 66.2 & 18.9 & 6.0 \\
\hline Centros de tratamiento de drogas & 84.5 & 86.6 & 47.4 & 68.0 \\
\hline Ámbito laboral (empresas) & 63.2 & 89.5 & 57.9 & 36.8 \\
\hline Comisarias (centros policiales) & 37.0 & 78.1 & 4.1 & 0.0 \\
\hline
\end{tabular}


Tabla 2

Descripción de los ítems del ASSIST

\begin{tabular}{|c|c|c|c|c|c|c|c|c|c|c|c|c|c|c|c|c|}
\hline & \multicolumn{4}{|c|}{ Tabaco $^{1}$} & \multicolumn{4}{|c|}{ Alcohol } & \multicolumn{4}{|c|}{ Marihuana } & \multicolumn{4}{|c|}{ Cocaína } \\
\hline & Media & $\mathrm{DE}$ & Asim. & Cur. & Media & DE & Asim. & Cur. & Media & $\mathrm{DE}$ & Asim. & Cur. & Media & $\mathrm{DE}$ & Asim. & Cur. \\
\hline Ítem 1 & 2.45 & 1.17 & -1.62 & 3.63 & 2.72 & 0.87 & -2.81 & 8.91 & 1.52 & 1.50 & -0.03 & 1.00 & 0.99 & 1.41 & 0.72 & 1.52 \\
\hline Ítem 2 & 3.73 & 2.58 & -0.52 & 1.53 & 2.71 & 1.71 & 0.06 & 2.57 & 1.79 & 2.10 & 0.76 & 2.27 & 2.76 & 2.42 & 0.13 & 1.49 \\
\hline Ítem 3 & 3.91 & 2.58 & -0.70 & 1.71 & 2.41 & 2.33 & 0.15 & 1.38 & 1.83 & 2.37 & 0.69 & 1.74 & 3.10 & 2.65 & -0.18 & 1.24 \\
\hline Ítem 4 & 0.51 & 1.61 & 2.99 & 10.50 & 1.07 & 2.17 & 1.66 & 4.02 & 0.52 & 1.58 & 2.87 & 9.90 & 2.77 & 3.00 & 0.25 & 1.23 \\
\hline Ítem 5 & - & - & - & - & 1.41 & 2.74 & 1.50 & 3.42 & 0.91 & 2.33 & 2.27 & 6.39 & 3.06 & 3.47 & 0.32 & 1.24 \\
\hline Ítem 6 & 1.87 & 2.63 & 0.81 & 1.80 & 1.58 & 2.44 & 1.06 & 2.35 & 1.23 & 2.25 & 1.44 & 3.34 & 3.16 & 2.77 & -0.11 & 1.19 \\
\hline Ítem 7 & 1.79 & 2.42 & 0.86 & 2.08 & 1.18 & 2.20 & 1.52 & 3.57 & 0.77 & 1.78 & 2.16 & 6.25 & 2.80 & 2.78 & 0.14 & 1.19 \\
\hline Total $^{2}$ & 1.99 & 5.64 & 2.81 & 9.49 & 2.17 & 6.62 & 3.56 & 14.99 & 0.74 & 3.71 & 6.05 & 42.58 & 1.74 & 6.94 & 4.00 & 17.78 \\
\hline
\end{tabular}

Nota. DE = Desviación Estándar; Asim. = Asimetría $;$ Cur. $=$ Curtosis

${ }^{1}$ El ítem 5 no se pregunta para tabaco

${ }^{2}$ El puntaje total por sustancia considera la sumatoría de las preguntas 2 a 7

rihuana y cocaína. Puede observarse que tanto para tabaco como para alcohol existe una mayor cantidad de respuestas positivas, la que disminuye para marihuana y para cocaína. Sin embrago, para esta última droga, las respuestas positivas (entre quienes respondieron positivamente en el ítem 1) fue mayor al de las demás sustancias analizadas.

De acurdo a los resultados del análisis factorial exploratorio se identificó un factor con valor propio mayor a 1 que, dependiendo la sustancia, explica entre un $60 \%$ y un $78 \%$ de la varianza.

\section{Consistencia interna}

La consistencia interna del ASSIST, tanto para el puntaje total como por sustancia específica fue adecuado en la muestra estudiada. El alpha de Cronbach para el puntaje total fue de .91 , para tabaco de .87 , alcohol .93 , marihuana .95 , cocaína .98 , anfetaminas .98 , inhalantes .90 , tranquilizantes .97 , alucinógenos .98 , y opiáceos .98 .

\section{Correlación test - retest}

Las correlaciones (CCI) para las cuatro sustancias con mayor prevalencia fueron: .63 (Intervalo de confianza del 95\% [IC95\%] .38 a $.87 ; F 7.08 ; p<.001)$ para tabaco .66 (IC95\% .46 - .86; F 7.33; $p<.001)$, para alcohol .74 (IC95\% .44 a $1.03 ; \mathrm{F} 11.81 ; p<.001)$ para marihuana y .80 (IC95\% .57 a $1.01 ; \mathrm{F} 12.35 ; p<.001)$ para cocaína-pasta base y cocaína. El resto de las sustancias no se mencionan debido su baja prevalencia en el retest (menor al 3\%).

\section{Comparación con Mini-Plus, abuso y dependencia}

La correlación (Pearson) entre el puntaje para dependencia ASSIST y el puntaje para el diagnóstico de síntomas de dependencia alcohol del MINI-Plus fue de $r=.66(p<$ .001). La correlación observada entre el puntaje para abuso del ASSIST y el puntaje para el diagnóstico de abuso del MINI-Plus fue de $r=.65(p<.001)$.

\section{Comparación de categoría de abuso o dependencia del MINI-Plus}

La comparación de medias de los puntajes específicos por sustancia ASSIST con la presencia o ausencia de signos de dependencia o abuso de alcohol (actual o durante la vida según el instrumento de medida MINI-Plus), presentó diferencias estadísticamente significativas entre ambos grupos, con una media mayor entre quienes presentaban abuso y/o dependencia (Tabla 3). No se analizaron las demás sustancias debido a su baja prevalencia en la muestra.

Tabla 3

Puntaje promedio del ASSIST según diagnóstico de abuso o dependencia

\begin{tabular}{|c|c|c|c|c|c|c|c|}
\hline \multirow[b]{3}{*}{$\begin{array}{l}\text { Puntaje } \\
\text { ASSIST }\end{array}$} & \multicolumn{7}{|c|}{ Categorías MINI-PLUS } \\
\hline & \multicolumn{2}{|c|}{ Presente } & \multicolumn{2}{|c|}{ Ausente } & \multirow[b]{2}{*}{$\mathbf{n}$} & \multirow{2}{*}{$\begin{array}{c}\text { t de } \\
\text { Student }\end{array}$} & \multirow[b]{2}{*}{ p-valor } \\
\hline & Media & $\begin{array}{c}\text { Desv. } \\
\text { típ. }\end{array}$ & Media & $\begin{array}{c}\text { Desv. } \\
\text { típ. }\end{array}$ & & & \\
\hline Tabaco & 15.18 & 8.33 & 11.07 & 8.55 & 326 & $-1.87^{*}$ & 0.03 \\
\hline Alcohol & 16.17 & 11.15 & 3.82 & 4.8 & 363 & $-13.82^{* * *}$ & $\leftarrow .001$ \\
\hline Marihuana & 14.13 & 7.29 & 5.98 & 9.1 & 203 & $-4.03 * * *$ & $\leftarrow .001$ \\
\hline Cocaína & 24.73 & 10.91 & 15.76 & 14.29 & 132 & $-2.98 * *$ & 0.001 \\
\hline
\end{tabular}

\section{Comparación con ASI-Lite. SDS. RTQ y AUDIT}

La correlación entre el puntaje del ASSIST y índice de severidad de adicción medido a través del ASI-Lite para las cuatro sustancia más prevalentes tuvo un rango entre .66 y $.83(p<.001)$. La correlación $r$ de Pearson entre el puntaje total del ASSIST y el puntaje total del instrumento SDS fue de $.65(p<.001)$. La correlación entre el puntaje para tabaco del ASSIST y el puntaje total del instrumento RTQ fue de $.60(p<.001)$. Por último, el puntaje total ASSIST para alcohol y el puntaje total del AUDIT fue de $r=.84(p<.001)$. 
Tabla 4

Resultado del análisis ROC de acuerdo a puntos de corte identificados.

\begin{tabular}{|c|c|c|c|c|c|c|c|c|c|}
\hline \multirow{2}{*}{$\begin{array}{l}\text { Riesgo } \\
\text { ASSIST }\end{array}$} & \multirow{2}{*}{$\begin{array}{c}\text { Puntaje } \\
\text { corte } \\
\text { ASSIST } \geq\end{array}$} & \multicolumn{6}{|c|}{ ROC } & \multirow[b]{2}{*}{ VPP \% } & \multirow[b]{2}{*}{ VPN \% } \\
\hline & & $A B C$ & IC $95 \%$ & $\begin{array}{c}\text { Sensibilidad } \\
\%\end{array}$ & $\begin{array}{c}\text { Especificidad } \\
\%\end{array}$ & $L R+$ & $L R-$ & & \\
\hline \multicolumn{10}{|l|}{ Tabaco } \\
\hline Intermedio & 4 & 0.93 & $(.90-.96)$ & 93.78 & 81.63 & 5.1 & 0.07 & 98.22 & 81.63 \\
\hline Alto & 21 & 0.77 & $(.59-.94)$ & 47.06 & 80 & 2.35 & 0.66 & 47.05 & 80 \\
\hline \multicolumn{10}{|l|}{ Alcohol } \\
\hline Intermedio & 11 & 0.88 & $(.84-.94)$ & 86.89 & 78.67 & 4.07 & 0.16 & 86.88 & 78.67 \\
\hline Alto & 21 & 0.77 & $(.66-.89)$ & 81.25 & 54.24 & 1.77 & 0.34 & 81.25 & 54.23 \\
\hline \multicolumn{10}{|l|}{ Marihuana } \\
\hline Intermedio & 4 & 0.93 & $(.89-.96)$ & 94.2 & 83.2 & 5.6 & 0.06 & 83.2 & 94.2 \\
\hline Alto & 21 & 0.88 & $(.79-.96)$ & 60 & 89.19 & 5.55 & 0.44 & 60 & 89.18 \\
\hline \multicolumn{10}{|l|}{ Cocaína } \\
\hline Intermedio & 4 & 0.94 & $(.89-.98)$ & 97.26 & 66.07 & 2.86 & 0.04 & 97.26 & 66.07 \\
\hline Alto & 21 & 0.74 & $(.58-.88)$ & 74.51 & 58.33 & 9.95 & 0.1 & 74.5 & 58.33 \\
\hline
\end{tabular}

Nota. $A B C=$ Área bajo la curva; $I C=$ Intervalo confianza; $L R+=$ Razón de verosimilitud positiva; $L R-=$ Razón de verosimilitud negativa; $V P P=$ Valor predictivo positivo; $V P N=$ Valor predictivo negativo.

\section{Validez discriminante del ASSIST}

En la tabla 4 se muestran los resultados del análisis ROC para tabaco, alcohol, marihuana y cocaína. El resto de las sustancias no se analizaron debido al insuficiente número de casos. La sensibilidad y especificidad para la distinción entre riesgo bajo y medio fue sobre el $80 \%$ con excepción de la especificidad para alcohol $(78.7 \%)$ y para cocaína $(66.1 \%)$.

La sensibilidad y especificidad del punto de corte propuesto por OMS para el riesgo alto ( $\geq 27$ puntos) fue de $7.8 \%$ y $93.3 \%$ para tabaco, $75.0 \%$ y $67.8 \%$ para alcohol, $35.0 \%$ y $100.0 \%$ para marihuana, y $60.8 \%$ y $87.5 \%$ para cocaína. En base a los resultados del análisis ROC se consideró un nuevo puntaje de corte ( $\geq 21$ puntos) que balanceara con mayor equilibrio entre sensibilidad y especificidad (ver tabla 4).

\section{Discusión}

El diseño y metodología para estudiar las evidencias de validez en Chile del instrumento de cribado ASSIST se orientaron principalmente en las validaciones multicéntricas realizadas por la OMS (Humeniuk et al., 2008) logrando verificar las propiedades psicométricas del instrumento. El ASSIST mostró una buena consistencia interna para cada sustancia y una buena correlación entre test y retest. La correlación entre el ASSIST y otros instrumentos (ASI-Lite, SDS, MINI-Plus, AUDIT y RTQ-Smoking) fue alta, entregando evidencia de validez concurrente de los puntajes. También hubo correspondencia en las categorías de abuso y dependencia entre los instrumentos de referencia y las categorías diagnósticas del ASSIST.
De acuerdo a los resultados del análisis factorial exploratorio se identificó un factor con valor propio mayor a 1 (entre 3,0 y 4,7 dependiendo la sustancia) y una alta carga factorial (>.7) para cada uno de los ítems incluidos. Este resultado confirma la existencia de un constructo -riesgo- que tiene diferentes niveles, y que es medido a través de 8 ítems que explora seis áreas relacionadas con el consumo de drogas. Finalmente los análisis ROC mostraron una adecuada sensibilidad y especificidad en relación al puntaje para definir riesgo intermedio definido por la OMS. Sin embargo, también se observó la necesidad de adecuar los puntos de corte para riesgo alto, principalmente por la baja sensibilidad del instrumento utilizando un punto de corte de 27 puntos para riesgo alto.

$\mathrm{Al}$ igual que en la validación original del instrumento (Humeniuk et al., 2008) la validez concurrente entre el ASSIST y otros instrumentos alcanzó correlaciones similares a los observados en el presente estudio, no obstante hubo divergencias respecto a la sensibilidad y especificidad con el puntaje de corte de alto riesgo que fue subsanado con puntajes de corte menores. En otro estudio que indagó las evidencias de validez del ASSIST en su adaptación al francés también se debió disminuir considerablemente los puntajes de corte de riesgo alto de uso de marihuana y alcohol para lograr una adecuada sensibilidad y especificidad (Khan et al., 2011).

Con las evidencias de validez del ASSIST en Chile se logra contar con un instrumento breve y útil para las estrategias de detección temprana del consumo de sustancia. El bajo costo de aplicación hace recomendable su uso en la atención primaria de salud, especialmente si se le vincula a una estrategia de intervención y referencia a tratamien- 
to (Humeniuk et al., 2008; Khan et al., 2012; Organización Mundial de la Salud, 2011; Monteiro, 2013).

El presente estudio tiene algunas limitaciones que deben ser tomadas en cuenta. En primer lugar, toda la información analizada se obtuvo a partir del auto informe de los participantes, lo cual pudiera distorsionar medidas como la intensidad, frecuencia o magnitud del consumo. No obstante lo anterior, la consistencia entre el test y el retest y la alta correlación con otros instrumentos entregan evidencia respecto a la validez de las puntuaciones del instrumento. En segundo lugar, no se alcanzó a un número suficiente de casos para realizar todas las prueba psicométricas en sustancias como anfetaminas, inhalantes, sedantes, alucinógenos y opiáceos, aunque esta es una situación similar a la ocurrida en otros estudios de validación del ASSIST (Khan et al., 2011; Newcombe et al., 2005). Por lo anterior, los puntajes de corte propuestos en este estudio pueden ser revisados en futuras investigaciones $y$, eventualmente, sufrir alguna modificación si hay suficiente evidencia que lo sustente.

Finalmente, los resultados del presente estudio indican que la versión chilena del instrumento ASSIST V.3 demostró evidencias de validez de sus puntuaciones para la identificación de distintos niveles de riesgo de consumo de alcohol, marihuana, tabaco y cocaína en población de diferentes ámbitos. Esta es la primera etapa de un programa de detección temprana, intervención breve y referencia a tratamiento que será implementado de manera piloto en 27 centros de atención primaria de salud para su posterior evaluación, revisión y escalamiento al nivel regional y nacional.

\section{Agradecimientos}

El estudio fue diseñado y financiado por el Servicio Nacional para la Prevención y Rehabilitación del Consumo de Drogas y Alcohol (SENDA) y conducido por el Laboratorio de Neurociencias Cognitiva y Social (LaNCyS) de la Facultad de Psicología de la Universidad Diego Portales. Agradecimientos especiales a los equipos de los centros de tratamiento, empresas y Carabineros de Chile que participaron en el estudio de validación.

\section{Conflicto de intereses}

Los autores declaran no tener conflicto de intereses.

\section{Referencias}

Alvarado, M. E., Garmendia, M. L., Acuña, G., Santis, R. y Arteaga, O. (2009). Validez y confiabilidad de la versión chilena del Alcohol Use Disorders Identification Test (AUDIT). Revista Médica de Chile, 137, 1463-1468.

Allen, J., Litten, R., Fertig, J. y Babor, T. (1997). A review of research on the Alcohol Use Disorders Identification Test (AUDIT). Alcoholism: Clinical and Experimental
Research, 21, 613 - 619. doi: 10.1111/j.1530-0277.1997. tb03811.x

American Psychiatric Association. (2000). Diagnostic and Statistical Manual of Mental Disorders. Fourth Edition. Text Revision (DSM-IV-TR). Arlington.

Babor, T. F. y Kadden, R. K. (2005). Screening and interventions for alcohol and drug problems in medical settings: what works? Journal of Trauma, 59, 80-87.

Bobes, J. (1998). A Spanish validation study of the mini international neuropsychiatric interview. European Psychiatry, 13, 198-199.

Bohn, M. J., Babor, T. F. y Kranzler, H. R. (1995). The Alcohol Use Disorders Identification Test (AUDIT): validation of a screening instrument for use in medical settings. Journal of Studies on Alcohol and Drugs, 56, 423-432.

Cacciola, J., Alterman, A., McLellan, A., Lin, Y. y Lynch, K. (2007). Initial evidence for the reliability and validity of a "Lite" version of the Addiction Severity Index. Drug and Alcohol Dependence, 87, 297-302. doi: 10.1016/j.drugalcdep.2006.09.002

Castillo-Carniglia, A., Kaufman, J. S., y Pino, P. (2013). Alcohol-attributable mortality and potential years of life lost in Chile in 2009. Alcohol and Alcoholism, 48, 729-36. doi: $10.1093 /$ alcalc/agt066

Cicchetti, D. V. y Sparrow, S. S. (1981). Developing criteria for establishing interrater reliability of specific items: Applications to assessment of adaptive behavior. American Journal of Mental Deficiency, 86, 127-137.

Clemente, M., Rubio, E., Pérez, A., Marrón, R., Herrero, I. y Fuertes, J. (2003). Determinación de la dependencia nicotínica en escolares fumadores a través de un test de Fagerström modificado. Anales de Pediatría, 58, 538-544.

Consejo Nacional para el Control de Estupefacientes. (2008). Validación Transcultural del Addiction Severity Index versión 6 (ASI v6). Chile. Informe final. Santiago: Consejo Nacional para el Control de Estupefacientes (CONACE). Ministerior del Interior. Escuela Salud Pública. Facultad de Medicina. Universidad de Chile.

Coulton, S., Drummond, C., James, D., Godfrey, C., Bland, J. M., Parrott, S. y Peters, T. (2006). Opportunistic screening for alcohol use disorders in primary care: comparative study. British Medical Journal, 332, 511-517. doi: 10.1136/bmj.38743.421574.7C

Departamento de Estadísticas e Información de Salud. (2013). Bases de datos Atenciones REM. 2011. Departamento de Estadísticas e Información de Salud de Chile (DEIS). Ministerio de Salud. Recuperado de http://deis.minsal. cl/BDPublica/BD_REM.aspx

Diaz, E., Garcia-Portilla, P., Saiz, P. A., Bobes Bascaran, T., Casares, M. J., Fonseca, E.,... Bobes, J. (2010). Rendimiento psicométrico de la sexta versión del Addiction Severity Index en español (ASI-6). Psicothema, 22, 513-519.

Drug Misuse Information Scotland. (2000). Integrated Care for Drug Users assessment Digest of Tools Used in 
the Assessment Process and Core Data Sets. Edinburgh: Effective Interventions Unit. Substance Misuse Division. Drug Misuse Information Scotland.

Fernandez, M. (2012). El costo socioeconómico del consumo de drogas ilícitas en Chile. Revista CEPAL, 107, 93-114.

Fondo Nacional de Salud. (2013). Distribución de la población beneficiaria respecto a la población total. regional y nacional. 2012. Fondo Nacional de Salud de Chile (FONASA). Recuperado de http://www.fonasa.cl/wps/ $\mathrm{wcm} /$ connect/internet/sa-general/informacion+corporativa/estadisticas+institucionales/estadisticas+institucionales

Gonzalez-Saiz, F., de Las Cuevas, C., Barrio, G. y Domingo-Salvany, A. (2008). Versión española consensuada de la Severity of Dependence Scale (SDS). Medicina Clínica, 131, 797-798.

Gossop, M., Best, D., Marsden, J. y Strang, J. (1997). Test-retest reliability of the Severity of Dependence Scale. Addiction, 92, 353.

Gossop, M., Darke, S., Griffiths, P., Hando, J., Powis, B., Hall, W. y Strang, J. (1995). The Severity of Dependence Scale (SDS): psychometric properties of the SDS in English and Australian samples of heroin. cocaine and amphetamine users. Addiction, 90, 607-614.

Hart, J. (2007). Correlation of health outcomes with physician and chiropractor ratios in the United States. Journal of Chiropractic Medicin, 6, 105-109

Henrique, I. F. S., De Micheli, D., Lacerda, R., Lacerda, L. y Formigoni, M. (2004). Validação da versão brasileira do teste de triagem do envolvimento com álcool. cigarro e outras substâncias (ASSIST). Revista da Associação Médica Brasileira, 50, 199-206. doi: 10.1590/S010442302004000200039

Hotham, E. (2009). Investigation of the alcohol. smoking and substance involment screening test (The ASSIST) in pregancy. Doctor of Philosophy. Faculty of Health Sciences. University of Adelaide. Adelaide. Australia.

Humeniuk, R., Ali, R., Babor, T., Farrell, M., Formigoni, M., Jittiwutikarn, J.,... Simon, S. (2008). Validation of the Alcohol. Smoking And Substance Involvement Screening Test (ASSIST). Addiction, 103, 1039 - 1047. doi: 10.1111/j.1360-0443.2007.02114

Iraurgi, I., González, F., Lozano, Ó., Landabaso, M. y Jiménez, J. (2010). Estimación de un punto de corte para la Escala de Severidad de la Dependencia (SDS) para la adicción a opiáceos mediante análisis ROC. Actas Españolas de Psiquiatría, 38, 270-277.

Iraurgi, I., Lozano, O., González-Saiz, F. y Trujols, J. (2008). Valoración psicométrica de la Escala de Severidad de la Dependencia a partir de dos modelos de análisis: la Teoría Clásica de los Test y la Teoría de Respuesta al Ítem. Boletín de Psicología, 93, 41-57.
Khan, R., Chatton, A., Nallet, A., Broers, B., Thorens, G., Achab-Arigo, S.,... Zullino, D. (2011). Validation of the French Version of the Alcohol. Smoking and Substance Involvement Screening Test (ASSIST). European Addiction Research, 17, 190 - 197. doi: 10.1159/000326073

Khan, R., Chatton, A., Thorens, G., Achab, S., Nallet, A., Broers, B.,... Khazaal, Y. (2012). Validation of the French version of the alcohol. smoking and substance involvement screening test (ASSIST) in the elderly. Substance Abuse Treatment. Prevention. and Policy, 7, 14. doi: 10.1186/1747-597X-7-14

Larrañaga, O. (1997). Eficiencia y equidad en el sistema de salud chileno. Santiago de Chile: Proyecto CEPAL/GTZ. Reformas financieras al sector salud en america latina y el caribe. Naciones Unidas.

Lecrubier, Y., Sheehan, D. V., Weiller, E., Amorim, P., Bonora, I., Harnett Sheehan, K.,... Dunbar, G. C. (1997). The Mini International Neuropsychiatric Interview (MINI). A short diagnostic structured interview: reliability and validity according to the CIDI. European Psychiatry, 12, 224-231. doi: 10.1016/S0924-9338(97)83296-8

Lorenzo-Seva, U., y Ferrando, P. J. (2006). FACTOR: A computer program to fit the exploratory factor analysis model. Behavior Research Methods, Instruments and Computers. 38, 88-91

McLellan, A., Luborsky, L., Woody, G. y O’Brien, C. (1980). An improved diagnostic evaluation instrument for substance abuse patients. The Addiction Severity Index. Journal of Nervous and Mental Disease, 168, 26-33.

Monteiro MG (2013). Alcohol y Salud Publica en América Latina: ¿cómo impedir un desastre sanitario?. Adicciones, 25, 99-105.

Newcombe, D., Humeniuk, R. y Ali, R. (2005). Validation of the World Health Organization Alcohol. Smoking and Substance Involvement Screening Test (ASSIST): report of results from the Australian site. Drug and Alcohol Review, 24, 217-226. doi: 10.1080/09595230500170266

Organización Mundial de la Salud. (2011). La prueba de detección de consumo de alcohol. tabaco y sustancias (ASSIST). Manual para uso en la atención primaria. Organización Mundial de la Salud. Ginebra.

Rubio, G., Bermejo, J., Caballero, M. y Santo-Domingo, J. (1998). Validacion de la prueba para la identificacion de trastornos por uso de alcohol (AUDIT) en Atencion Primaria. Revista Clínica Española, 198, 11-14.

Saunders, J., Aasland, O., Babor, T., de la Fuente, J. y Grant, M. (1993). Development of the Alcohol Use Disorders Identification Test (AUDIT): WHO Collaborative Project on Early Detection of Persons with Harmful Alcohol Consumption. Addiction, 88, 791-804. doi: 10.1111/ j.1360-0443.1993.tb02093.x

Servicio Nacional para la Prevención y Rehabilitación del Consumo de Drogas y Alcohol. (2013). Décimo Estudios Nacional de Drogas en Población Genera de Chile. Santiago. 
Sheehan, D., Lecrubier, Y., Sheehan, K., Amorim, P., Janavs, J., Weiller, E.,... Dunbar, G. (1998). The Mini-International Neuropsychiatric Interview (M.I.N.I.): the development and validation of a structured diagnostic psychiatric interview for DSM-IV and ICD-10. Journal of Clinical Psychiatry, 59, 22-57.

StataCorp. (2009). Stata Statistical Software: Release 11. College Station, TX: StataCorp LP

Tate, J. y Schmitz, J. (1993). A proposed revision of the Fagerstrom Tolerance Questionnaire. Addictive Behaviors, 18, 135-143. doi: 10.1016/0306-4603(93)90043-9

Watson, J., Crosby, H., Dale, V., Tober, G., Wu, Q., Lang, J.,... Coulton, S. (2013). AESOPS: a randomised controlled trial of the clinical effectiveness and cost-effectiveness of opportunistic screening and stepped care interventions for older hazardous alcohol users in primary care. Health Technology Assessment Journal, 17, 1-158. doi: $10.3310 /$ hta17250

WHO ASSIST Working Group. (2002). The Alcohol. Smoking and Substance Involvement Screening Test (ASSIST): development. reliability and feasibility. Addiction, 97, 1183-1194. doi: 10.1046/j.1360-0443.2002.00185.x

World Health Organization. (2010). Atlas on substance use. Resources for the prevention and treatment of substance use disorders. Geneva World Health Organization. 


\section{Apéndice: Versión final ASSIST-Chile}

OMS - ASSIST V3.0 - CHILE

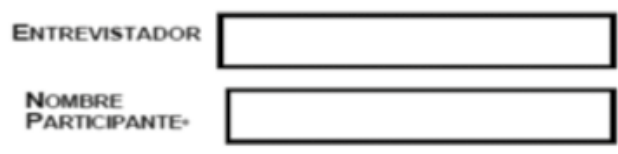
CONTEXTO
O LUGAR NOMBRE

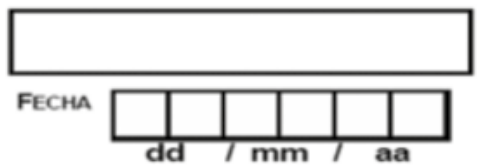

Pregunta 1 MOSTRAR TARJETA DE DROGAS $N^{\circ} 1$

Alguna vez en su vida, ¿Ha
consumido alguna de las siguientes
sustancias? (SIN RECETA O
INDICACIÖN MEDICA)

\begin{tabular}{|l|ll|}
\hline \multicolumn{1}{|l|}{$\begin{array}{l}\text { Alguna vez en su vida, ¿Ha } \\
\text { consumido alguna de las siguientes } \\
\text { sustancias? (SIN RECETA O } \\
\text { INDICACIÖN MÉDICA) }\end{array}$} & No & Si \\
\hline a. Tabaco & 0 & 3 \\
\hline b. Bebidas alcohólicas & 0 & 3 \\
\hline c. Marihuana & 0 & 3 \\
\hline d. Cocaina & 0 & 3 \\
\hline e. Anfetaminas u otro tipo de estimulantes & 0 & 3 \\
\hline f. Inhalantes & 0 & 3 \\
\hline g. Tranquilizantes o pastillas para dormir & 0 & 3 \\
\hline h. Alucinógenos & 0 & 3 \\
\hline i. Opiáceos & 0 & 3 \\
\hline j. Otros - especifique: & 0 & 3 \\
\hline
\end{tabular}

1.2 Compruebe si todas las respuestas son negativas: "¿Tampoco cuando iba al colegio?"
Pregunta 2 MUESTRE TARJETA DE RESPUESTA N ${ }^{\circ} 2$

\begin{tabular}{|c|c|c|c|c|c|}
\hline $\begin{array}{l}\text { En los últimos } 3 \text { meses ¿Con qué } \\
\text { frecuencia ha consumido: (PRIMERA } \\
\text { DROGA, SEGUNDA DROGA, ETC)? }\end{array}$ & $\frac{3}{2}$ & 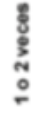 & $\frac{1}{2}$ & $\begin{array}{l}\text { हू } \\
\text { E् } \\
\text { है } \\
\text { है }\end{array}$ & 象 \\
\hline a. Tabaco & 0 & 2 & 3 & 4 & 6 \\
\hline b. Bebidas alcohólicas & 0 & 2 & 3 & 4 & 6 \\
\hline c. Marihuana & 0 & 2 & 3 & 4 & 6 \\
\hline d. Cocaina & 0 & 2 & 3 & 4 & 6 \\
\hline e. Anfetaminas u otro tipo de estimulantes & 0 & 2 & 3 & 4 & 6 \\
\hline f. Inhalantes & 0 & 2 & 3 & 4 & 6 \\
\hline g. Tranquilizantes o pastillas para dormir & 0 & 2 & 3 & 4 & 6 \\
\hline h. Alucinógenos & 0 & 2 & 3 & 4 & 6 \\
\hline i. Opiáceos & 0 & 2 & 3 & 4 & 6 \\
\hline j. Otros - especifique: & 0 & 2 & 3 & 4 & 6 \\
\hline
\end{tabular}

Si ha respondido "Nunca" a todos los items on la Progunta 2, salte a la Progunta 6, do los contrario continúe con la entrevista.

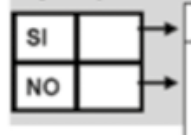
Vuelva a leer la Pregunta 1
si contestó "No" a todos los items, pare la
entrovista.
-Si contesto "SI" a alguno do los items de la P1, siga a la
P2 bara cada sustancia aue hava consumido alauna vez.

Pregunta 3

\begin{tabular}{|c|c|c|c|c|c|}
\hline $\begin{array}{l}\text { En los últimos tres meses, ¿con qué } \\
\text { frecuencia ha tenido fuertes deseos o } \\
\text { ansias de consumir (PRIMERA DROGA, } \\
\text { SEGUNDA DROGA, ETC)? }\end{array}$ & $\frac{5}{\frac{5}{2}}$ & 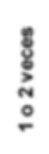 & 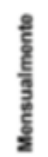 & $\begin{array}{l}\text { हूँ } \\
\text { हूँ } \\
\text { हूँ } \\
\text { हू }\end{array}$ & 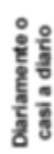 \\
\hline a. Tabaco & 0 & 3 & 4 & 5 & 6 \\
\hline b. Bebidas alcohólicas & 0 & 3 & 4 & 5 & 6 \\
\hline c. Marihuana & 0 & 3 & 4 & 5 & 6 \\
\hline d. Cocaina & 0 & 3 & 4 & 5 & 6 \\
\hline e. Anfetaminas u otro tipo de estimulantes & 0 & 3 & 4 & 5 & 6 \\
\hline f. Inhalantes & 0 & 3 & 4 & 5 & 6 \\
\hline g. Tranquilizantes o pastillas para dormir & $\mathbf{0}$ & 3 & 4 & 5 & 6 \\
\hline h. Alucinógenos & 0 & 3 & 4 & 5 & 6 \\
\hline i. Opiáceos & 0 & 3 & 4 & 5 & 6 \\
\hline j. Otros - especifique: & 0 & 3 & 4 & 5 & 6 \\
\hline
\end{tabular}

\section{Pregunta 4}

En los últimos tres meses, ¿con qué

frecuencia ha tenido problemas que

sociales, legales o económicos debido al

consumo de: (PRIMERA DROGA,

SEGUNDA DROGA, ETC)?

\begin{tabular}{|l|lllll|}
\hline a. Tabaco & 0 & 4 & 5 & 6 & 7 \\
\hline b. Bebidas alcoholicas & 0 & 4 & 5 & 6 & 7 \\
\hline c. Marihuana & 0 & 4 & 5 & 6 & 7 \\
\hline d. Cocaina & 0 & 4 & 5 & 6 & 7 \\
\hline e. Anfetaminas u otro tipo de estimulantes & 0 & 4 & 5 & 6 & 7 \\
\hline f. Inhalantes & 0 & 4 & 5 & 6 & 7 \\
\hline g. Tranquilizantes o pastillas para dormir & 0 & 4 & 5 & 6 & 7 \\
\hline h. Alucinógenos & 0 & 4 & 5 & 6 & 7 \\
\hline i. Opiáceos & 0 & 4 & 5 & 6 & 7 \\
\hline j. Otros - especifique: & 0 & 4 & 5 & 6 & 7 \\
\hline
\end{tabular}

Tavieta de respuesta - sustancias

a. Tabaco (cigarrillos, cigarros habanos, tabaco de mascar, pipa, etc.)

b. Bebidas alcohólicas (cerveza, vino, licores, destilados, etc.)

c. Marihuana (cannabis, pitos, hierba, hashish, etc.)

d. Cocaina (coca, pasta base, falopa, crack, etc.)

e. Antetaminas u otro tipo de estimulantes (speed, éxtasis, pldoras adelgazantes, cristal, etc.)

f. Inhalantes (neoprén, gasolina/bencina, pegamentos, solventes, poper, aerosoles, etc.)

9. Tranquilicantes o pastilas para dormir (valium/diazepam, RavotrivClonazepam, Alprazolam, Amparaxlorazepam, Zopiclona, Dormonid/Midazolam, chicotaltunitrazepam, etc)

h. Alucinógenos (LSD, ácidos, hongos, peyote, ketamina, PCP, etc.)

i. Opiáceos (codeina/arabes, Tramal/Tramadol, morfina, metadona, heroina, petidina, ete.)

1. Otros - especifique: (por ejemplo relajantes/sedantes, modafinilo/Mentix, esteroides, anabólicos, etc.) 


\section{Pregunta 5}

\begin{tabular}{|c|c|c|c|c|c|}
\hline $\begin{array}{l}\text { En los últimos tres meses, ¿con qué } \\
\text { frecuencia ha tenido problemas para } \\
\text { cumplir con sus obligaciones habituales } \\
\text { a causa del consumo de (PRIMERA } \\
\text { DROGA, SEGUIIDA DROGA, ETC)? }\end{array}$ & $\frac{\tilde{g}}{2}$ & \begin{tabular}{l} 
8. \\
\multirow{2}{*}{} \\
$\stackrel{2}{\circ}$
\end{tabular} & 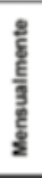 &  & 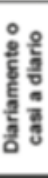 \\
\hline \multicolumn{6}{|l|}{ a. Tabaco } \\
\hline b. Bebidas alcohólicas & 0 & 5 & 6 & 7 & 8 \\
\hline c. Marihuana & 0 & 5 & 6 & 7 & 8 \\
\hline d. Cocaina & 0 & 5 & 6 & 7 & 8 \\
\hline e. Anfetaminas u otro tipo de estimulantes & 0 & 5 & 6 & 7 & 8 \\
\hline f. Inhalantes & 0 & 5 & 6 & 7 & 8 \\
\hline g. Tranquilizantes o pastillas para dormir & 0 & 5 & 6 & 7 & 8 \\
\hline h. Alucinógenos & 0 & 5 & 6 & 7 & 8 \\
\hline i. Opiáceos & 0 & 5 & 6 & 7 & 8 \\
\hline j. Otros - especifique: & 0 & 5 & 6 & 7 & 8 \\
\hline
\end{tabular}

\section{Pregunta 7}

\begin{tabular}{|c|c|c|c|}
\hline $\begin{array}{l}\text { ¿Alquna vez ha intentado controlar, } \\
\text { reducir o dejar de consumir } \\
\text { (PRIMERA DROGA, SEGUIIDA DROGA, } \\
\text { ETC) y no lo ha logrado? }\end{array}$ & 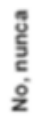 & 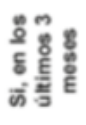 & 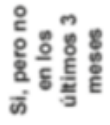 \\
\hline a. Tabaco & 0 & 6 & 3 \\
\hline b. Bebidas alcoholicas & 0 & 6 & 3 \\
\hline c. Marihuana & 0 & 6 & 3 \\
\hline d. Cocaina & 0 & 6 & 3 \\
\hline e. Anfetaminas u otro tipo de estimulantes & 0 & 6 & 3 \\
\hline f. Inhalantes & 0 & 6 & 3 \\
\hline g. Tranquilizantes o pastillas para dormir & 0 & 6 & 3 \\
\hline h. Alucinogenos & 0 & 6 & 3 \\
\hline i. Opiáceos & 0 & 6 & 3 \\
\hline j. Otros - especifique: & 0 & 6 & 3 \\
\hline
\end{tabular}

Haga las preguntas 6 y 7 para todas las sustancias que ha
consumido alguna vez (es decir, aquellas abordadas en la Pregunta 1)

\begin{tabular}{|c|c|c|c|}
\hline $\begin{array}{l}\text { ¿Alguna vez amigos, familiares } \\
\text { alguien más le han mostrado } \\
\text { preocupación por su consumo de: } \\
\text { (PRIMERA DROGA, SEGUIIDA DROGA, } \\
\text { ETC)? }\end{array}$ & 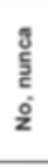 & 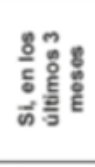 & 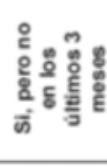 \\
\hline a. Tabaco & 0 & 6 & 3 \\
\hline b. Bebidas alcoholicas & 0 & 6 & 3 \\
\hline c. Marihuana & 0 & 6 & 3 \\
\hline d. Cocaina & 0 & 6 & 3 \\
\hline e. Anfetaminas u otro tipo de estimulantes & 0 & 6 & 3 \\
\hline f. Inhalantes & 0 & 6 & 3 \\
\hline g. Tranquilizantes o pastillas para dormir & 0 & 6 & 3 \\
\hline h. Alucinógenos & 0 & 6 & 3 \\
\hline i. Opiáceos & 0 & 6 & 3 \\
\hline j. Otros - especifique: & 0 & 6 & 3 \\
\hline
\end{tabular}

\section{COMO CAL CULAR UNA PUNTUACICN ESPECCIACA PARA CADA SUSTANCIA}

Para cada sustancia (etiquetadas de la a. la j.) sume las puntuaciones de las preguntas 2 a la 7 , ambas inclusive. No incluya los resultados ni de la pregunta 1 ni de la 8 en esta puntuación. Por ejemplo, la puntuación para el cannabis se calcula como: $\mathrm{P} 2 \mathrm{C}+\mathrm{P} 3 \mathrm{C}+\mathrm{P} 4 \mathrm{c}+\mathrm{P} 5 \mathrm{c}+\mathrm{P} 6 \mathrm{c}+\mathrm{P} 7 \mathrm{c}$

Note que la P5 para el tabaco no está codificada y se calcula como: P2a + P3a + P4a + P6a + P7a

EL TIPO DE INTERVENCICN SE DETERMENA POR LA PUNTUACION ESPECIFCA DEL PACIENTE PARA CADA SUSTANCIA

\begin{tabular}{|l|c|c|c|c|}
\hline & $\begin{array}{c}\text { Registre la puntuación para sustancia } \\
\text { especifica }\end{array}$ & Intervención Mínima & Intervención Breve & Tratamiento más intensivo * \\
\hline a. tabaco & & $0-3$ & $4-26$ & $27+$ \\
\hline b. alcohol & & $0-10$ & $11-26$ & $27+$ \\
\hline c. marihuana & & $0-3$ & $4-26$ & $27+$ \\
\hline d. cocaína & & $0-3$ & $4-26$ & $27+$ \\
\hline e. anfetaminas & & $0-3$ & $4-26$ & $27+$ \\
\hline f. inhalantes & & $0-3$ & $4-26$ & $27+$ \\
\hline g. sedantes & & $0-3$ & $4-26$ & $27+$ \\
\hline h. alucinógenos & & $0-3$ & $4-26$ & $27+$ \\
\hline i. opiáceos & & $0-3$ & $4-26$ & $27+$ \\
\hline j. otras drogas & & $0-3$ & 27 \\
\hline
\end{tabular}

NOTA: "UNA MAYOR EVALUACION Y TRATAMENTO MAS INTENSNO puede ser proporcionado por profesionales sanitarios capacitados en adicciones dentro del ámbito de Atención Primaria, o por un servicio especializado para las adicciones cuando esté disponible. 